\title{
Optical control and switching of excitation transfer in nano-arrays
}

\author{
David S. Bradshaw and David L. Andrews* \\ Nanostructures and Photomolecular Systems, School of Chemical Sciences, \\ University of East Anglia, Norwich NR4 7TJ, U.K.
}

\begin{abstract}
The possibility of influencing resonance energy transfer through the input of off-resonant pulses of laser radiation is the subject of recent research. Attention is now focused on systems in which resonance energy transfer is designedly precluded by geometric configuration. Here, through an optically nonlinear mechanism - optically controlled resonance energy transfer - the throughput of non-resonant pulses can facilitate energy transfer that is, in their absence, completely forbidden. The system thus functions as an optical buffer, with excitation throughput switched on by the secondary beam. For applications, a system based on two parallel nano-arrays is envisaged. This paper will establish and discuss the principles - those that can be exploited to enhance switching characteristics and efficiency, and others (such as offaxis excitation transfer) that may represent cross-talk limitations. Principles to be explored in detail are the interplay between geometric features, including the array architecture and repeat distance (lattice constant), the array spacing and translational symmetry, the orientations of the transition dipoles, and the magnitude of the relevant components of the nonlinear response tensors. The aim is, through a determination of key parameters, to inform a program of optimization that can deliver specific criteria for realizing the most efficient systems for implementation.
\end{abstract}

Keywords: Nanophotonics, energy transfer, quantum electrodynamics, near-field, optical transistor, optical switching, nano-array

\section{INTRODUCTION}

As the speed of ultrafast communications, sensing and computer processing maintains an apparently unabated rise, the need to achieve ever faster switching capability becomes increasingly pressing. Although electronics capabilities generally continue to improve according to Moore's Law, all-optical systems have a clear potential for much greater bandwidths and speeds - circumventing the bottlenecks that can often result from opto-electronic conversion. For many years it has been known that all-optical switching, based on various forms of photonic interaction in which light is controlled by light, is not only technically realizable but has the potential to revolutionize telecommunications and computing. Not surprisingly, numerous implementation strategies have been entertained, and many are the subject of vigorous ongoing research. This paper presents an analysis of a novel optical control mechanism - initially outlined by one of the present authors. ${ }^{1-3}$

Resonance energy transfer is the principal process for the intermolecular redistribution of electronic energy following initial (usually optical) excitation. ${ }^{4}$ In its simplest form known as Förster transfer, it involves a simple relocation of energy from an electronically excited donor $A$ to an acceptor $B$ in its ground state. By input of an auxiliary laser field this energy transfer may be enhanced by a laser-assisted resonance energy transfer (LARET) mechanism, whose efficiency scales linearly with the laser intensity. ${ }^{5}$ Calculations have shown that LARET can offer a several-fold increase in the rate of energy transfer, even for modest pulsed laser intensities of around $10^{12} \mathrm{~W} \mathrm{~cm}^{-2}{ }^{6} \mathrm{Optically}$ controlled resonance energy transfer (OCRET) is a related process, differing from LARET in that an important configurable condition is applied. This condition requires the transition dipole moments of the donor and acceptor, and their mutual displacement vector, to be mutually perpendicular - thus excluding a Förster process that would otherwise

*david.andrews@physics.org 


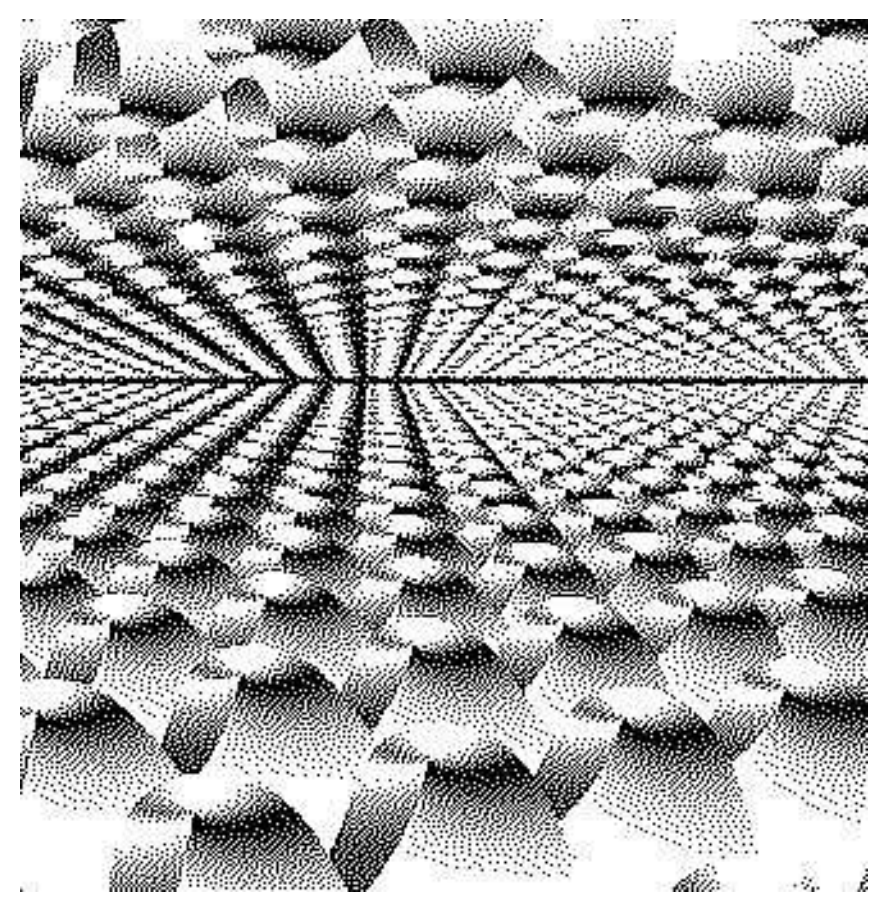

Figure 1. Graphic depiction of a parallel pair of two-dimensional hexagonal-lattice arrays

be possible on symmetry and energetic grounds. ${ }^{7}$ By application of the off-resonant laser beam the transfer of energy is activated, effecting all-optical switching action.

In the following we develop the fundamental electrodynamic theory for the OCRET mechanism, identifying in detail the geometric, optical and molecular structural conditions for it to occur between a single donor-acceptor pair (Section 2). The analysis is then extended to several arrangements - one of which is figuratively illustrated by Fig. 1 that could be useful for realistic all-optical switches, taking into account the requirement for a multiplicity of such donoracceptor partners to operate independently, without significant cross-talk. The specific geometric configurations to be examined are one-dimensional linear arrays (Section 3), two-dimensional square-lattice arrays (Section 4) and hexagonal-lattice arrays (Section 5). Further implementation issues are addressed in the concluding Section 6.

\section{COUPLING PAIR}

To specifically determine the rate of energy transfer for the OCRET mechanism, a time-dependent perturbation theory method is required. Details of the calculation, which is used to establish the quantum amplitudes of the system, are fully described in refs 5 and 6 and they are not repeated here. The OCRET mechanism is distinguished by the fact that the quantum amplitude for Förster transfer has a null result - a consequence of the orthogonal triad condition, i.e. $\mathbf{R} \perp \boldsymbol{\mu}_{A} \perp \boldsymbol{\mu}_{B}$, where $\mathbf{R}$ is the donor-acceptor displacement vector, and $\boldsymbol{\mu}_{A}, \boldsymbol{\mu}_{B}$ are the salient transition dipole moments of the two molecules. ${ }^{8}$ In the near-field, the quantum amplitude arising from the input auxiliary beam is given by;

$$
M_{f i}^{(4)}=\left(\frac{n \hbar c k}{8 \pi \varepsilon_{0}^{2} V R^{3}}\right) e_{i} \bar{e}_{l}\left(\delta_{j k}-3 \hat{R}_{j} \hat{R}_{k}\right)\left(S_{i j}^{A}(k) S_{l k}^{B}(-k)+S_{i j}^{B}(k) S_{l k}^{A}(-k)\right),
$$

where $n$ is the number of photons (proportional to intensity) in the quantization volume $V$, and the implied summation 


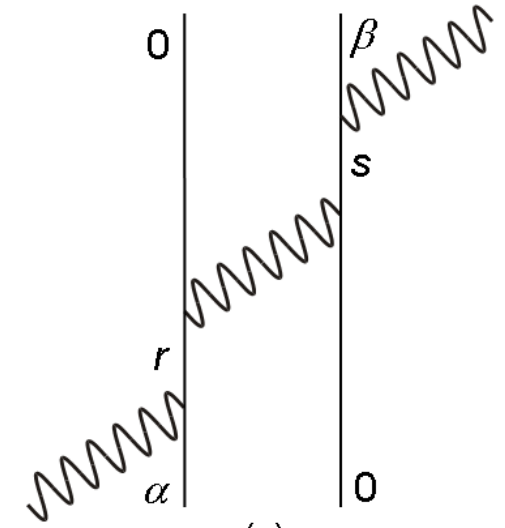

(a)

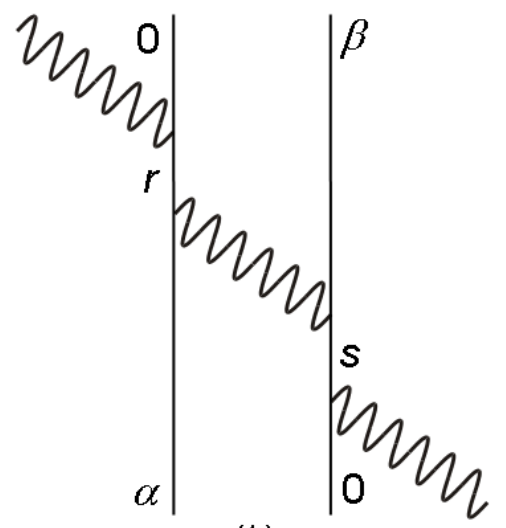

(b)

Figure 2. Feynman diagrams for OCRET

convention for repeated Cartesian tensor indices is employed. Furthermore, e and $\hbar c k$ denote the polarization vector (an overbar denoting complex conjugation) and energy of the input photon, respectively. Each of the two terms of equation (1), illustrated by the Feynman diagrams of Fig. 2, represents a fourth order photonic interaction event, necessitating deployment of a fourth-order perturbation treatment as indicated by the superscript of $M_{f i}$. Also in (1) is the generalized polarizability, $S_{i j}^{\xi}( \pm k)$, explicitly given by;

$$
S_{i j}^{\xi}( \pm k)=\sum_{r}\left\{\frac{\mu_{i}^{f r} \mu_{j}^{r i}}{\tilde{E}_{r f} \pm \hbar c k}+\frac{\mu_{j}^{f r} \mu_{i}^{r i}}{\tilde{E}_{r i} \mp \hbar c k}\right\}
$$

where the transition dipole moments are designated by the shorthand notation $\boldsymbol{\mu}^{x y}=\langle x|\boldsymbol{\mu}| y\rangle$, energy differences are expressed in the form $E_{x y}=E_{x}-E_{y}$, and tildes denote the necessary inclusion of damping terms if the theory were to be applied under near-resonance conditions ${ }^{9-15}$ - though the present analysis focuses solely on off-resonant photon absorption, and as such the tildes will henceforth be omitted. Further, $f$ signifies the final electronic state of molecule $\xi, i$ is the initial state, and $r, s$ are intermediates. To a good approximation, let it be assumed that the sums of equation (2) are limited to the three states that determine the most prominent optical features. These are denoted $|0\rangle,|\sigma\rangle,|\alpha\rangle$ for donor $A$, and $|0\rangle,|\tau\rangle,|\beta\rangle$ for acceptor $B$ - where $|\alpha\rangle$ and $|\beta\rangle$ are the levels between which energy transfer occurs. It is also expedient to select a frequency for the input radiation that has a resonance offset with respect to the positioning of these levels, a condition expressible as $E_{\sigma \alpha}=\hbar c k+\Delta E_{A}$, where $\Delta E_{A}$ is a non-zero energy with magnitude significantly lower than a typical transition energy. An expression of similar form, $E_{\tau \beta}=\hbar c k+\Delta E_{B}$, is assumed for $B$. The outcome of applying these conditions is that one summand is significantly larger in magnitude than the rest. Hence, equation (1) becomes;

$$
M_{f i}^{(4)}=\left(\frac{n \hbar c k\left(\mathbf{e} \cdot \boldsymbol{\mu}^{\sigma \alpha}\right)\left(\overline{\mathbf{e}} \cdot \boldsymbol{\mu}^{\beta \tau}\right)}{8 \pi \varepsilon_{0}^{2} V R^{3} \Delta E_{A} \Delta E_{B}}\right)\left(\boldsymbol{\mu}^{0 \sigma} \cdot \boldsymbol{\mu}^{\tau 0}-3\left(\boldsymbol{\mu}^{0 \sigma} \cdot \hat{\mathbf{R}}\right)\left(\boldsymbol{\mu}^{\tau 0} \cdot \hat{\mathbf{R}}\right)\right) .
$$

Next, to proceed to the geometric conditions, we introduce a Cartesian basis in which the donor-acceptor displacement vector $\mathbf{R}$ is identified with the $z$-direction. The vectors $\hat{\boldsymbol{\mu}}_{A} \equiv \hat{\boldsymbol{\mu}}^{0 \alpha}$ and $\hat{\boldsymbol{\mu}}_{B} \equiv \hat{\boldsymbol{\mu}}^{\beta 0}$ are chosen unambiguously as being directed in the $\hat{\mathbf{i}}$ and $\hat{\mathbf{j}}$ directions respectively and, by a judicious choice which is fully justifiable on symmetry grounds, $\hat{\boldsymbol{\mu}}^{0 \sigma}=\hat{\mathbf{k}}$ and $\hat{\boldsymbol{\mu}}^{\sigma \alpha}=\hat{\mathbf{j}}$. Due to the mutually orthogonal triad of vectors that determines the conditions for OCRET, the dipole orientations of the acceptor are easily determined by taking these vectors and rotating each by $90^{\circ}$ around the $z$-axis, so that $\hat{\boldsymbol{\mu}}^{\tau 0}=\hat{\mathbf{k}}$ and $\hat{\boldsymbol{\mu}}^{\beta \tau}=-\hat{\mathbf{i}}$. For convenience $A$ and $B$ are chosen to belong to identical symmetry groups, although the same calculational method allows for systems where this is not necessarily the case. Therefore, employing this configuration, equation (3) becomes; 


$$
M_{f i}^{(4)}=\left(\frac{-n \hbar c k|\boldsymbol{\mu}|^{4} \kappa^{\prime}}{8 \pi \varepsilon_{0}^{2} V r^{3} \Delta E_{A} \Delta E_{B}}\right) \sin ^{2} \phi \cos \theta \sin \theta,
$$

where the orientation factor is $\kappa^{\prime}=\left(R^{2}-3 r^{2}\right) / R^{2}=-2$ given that $\mathbf{R}=r \hat{\mathbf{k}}, r$ being the displacement of $B$ from $A$. Furthermore, the given angles denote the orientation of $\mathbf{e}$ in spherical coordinates (with the input photon polarization taken as linear). In addition, all transition dipole moments will have similar magnitude - thus, for simplicity, assumed to be equal. Equation (4) clearly delivers a non-zero result, unlike the now precluded Förster transfer. The time-dependent probability, $P(t)$, that the energy transfer process can proceed is secured from the Fermi's Golden Rule, ${ }^{16}$ here expressible in the form;

$$
P(t)=\frac{2 \pi \rho_{f}}{\hbar} \int_{0}^{t}\left|M_{f i}^{(2)}+M_{f i}^{(4)}+\ldots\right|^{2} \mathrm{~d} t
$$

with higher-order quantum amplitudes rapidly diminishing in magnitude. Here, the second-order contribution $M_{f i}^{(2)}$ corresponds to RET - a null quantity in this case - and $\rho_{f}$ is the density of states. Inserting (4) into (5) gives the expression;

$$
P(t)=\frac{4 K C^{2} J^{\prime}}{r^{6}} \sin ^{4} \phi \cos ^{2} \theta \sin ^{2} \theta
$$

where the variables are defined as $K=|\boldsymbol{\mu}|^{4} \rho_{f} / 8 \pi \varepsilon_{0}^{2} \hbar, \quad C=|\boldsymbol{\mu}|^{2} / 2 c \varepsilon_{0} \Delta E_{A} \Delta E_{B}$ and $\quad J^{\prime}=\int_{0}^{t} I^{2}(t) \mathrm{d} t$, in which $I(t) \equiv n \hbar c^{2} k / V$ is the irradiance. It is noteworthy that with $\phi=0^{\circ}, \theta=0^{\circ}$ or $\theta=90^{\circ}$, no energy transfer to the acceptor occurs.

\section{ONE-DIMENSIONAL LINEAR ARRAYS}

To extend and develop the pair model of the last section into that of a potentially workable all-optical switch, we shall initially investigate the case of coupling between two one-dimensional linear arrays - each of which is composed of equally spaced, identical molecules. These are constructed such that each constituent molecule of the donor array directly corresponds to a counterpart within the acceptor array; these pairs are given the coordinate $u$ (an integer value) and are displaced, one from each other, by $r \hat{\mathbf{k}}$. Furthermore, all molecules in the donor and acceptor arrays are orientated in the $\hat{\mathbf{i}}$ and $\hat{\mathbf{j}}$ directions, respectively, i.e. the principal condition of OCRET is satisfied. This linear array system is equivalent to a single row of Fig. 3. It is expedient to focus on processes by means of which energy can transfer from any one specific excited donor (for convenience located at $u=0$ ) to any other molecule. First we consider energy relocation to any single, arbitrary molecule within the acceptor array. To find the relevant quantum amplitude, the same mathematical process is utilized as the preceding section up to equation (4) when, as $\mathbf{R}=u \hat{\mathbf{i}}+r \hat{\mathbf{k}}$ in this instance (where $l$ is the lattice constant), we find;

$$
M_{f i}^{(4)}=\left(\frac{-n \hbar c k|\boldsymbol{\mu}|^{4}}{8 \pi \varepsilon_{0}^{2} l^{3} V \Delta E_{A} \Delta E_{B}}\right)\left(\frac{u^{2}-2 r^{\prime 2}}{\left(u^{2}+r^{\prime 2}\right)^{\frac{5}{2}}}\right) \sin ^{2} \phi \cos \theta \sin \theta,
$$

since $\kappa^{\prime}=\left(u^{2}-2 r^{\prime 2}\right) /\left(u^{2}+r^{\prime 2}\right)$, in which $r^{\prime}=r / l$ is the aspect ratio. As anticipated it is clear that equation (7) is 


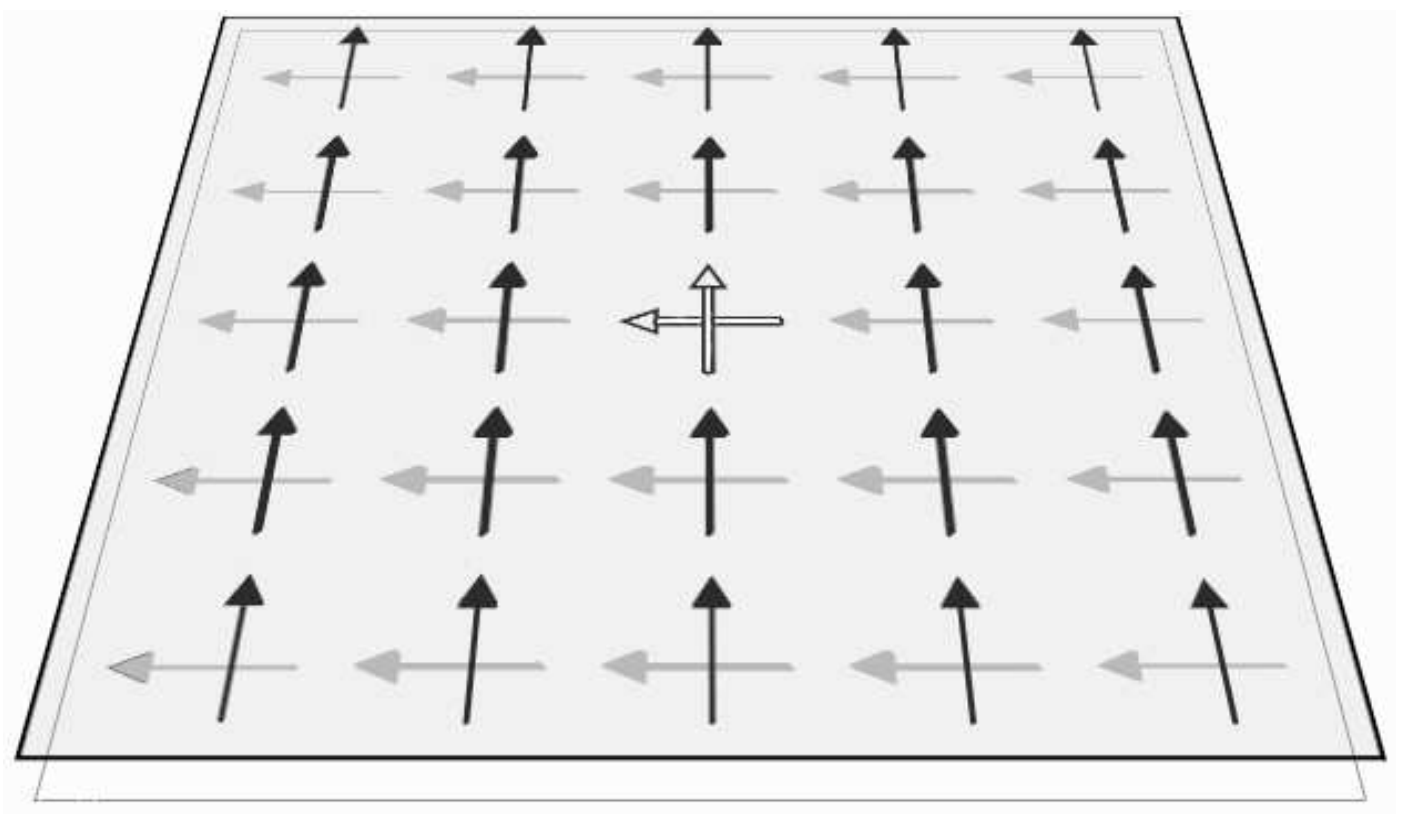

Figure 3. Structure of the parallel arrays, viewed from above. Both lie in the $i j$-plane, with all donor transition moments (black) in the upper array parallel to the $i$-axis, and all acceptor transition moments (gray) in the lower array parallel to the $j$-axis.

The open arrows represent one excited donor and its counterpart acceptor.

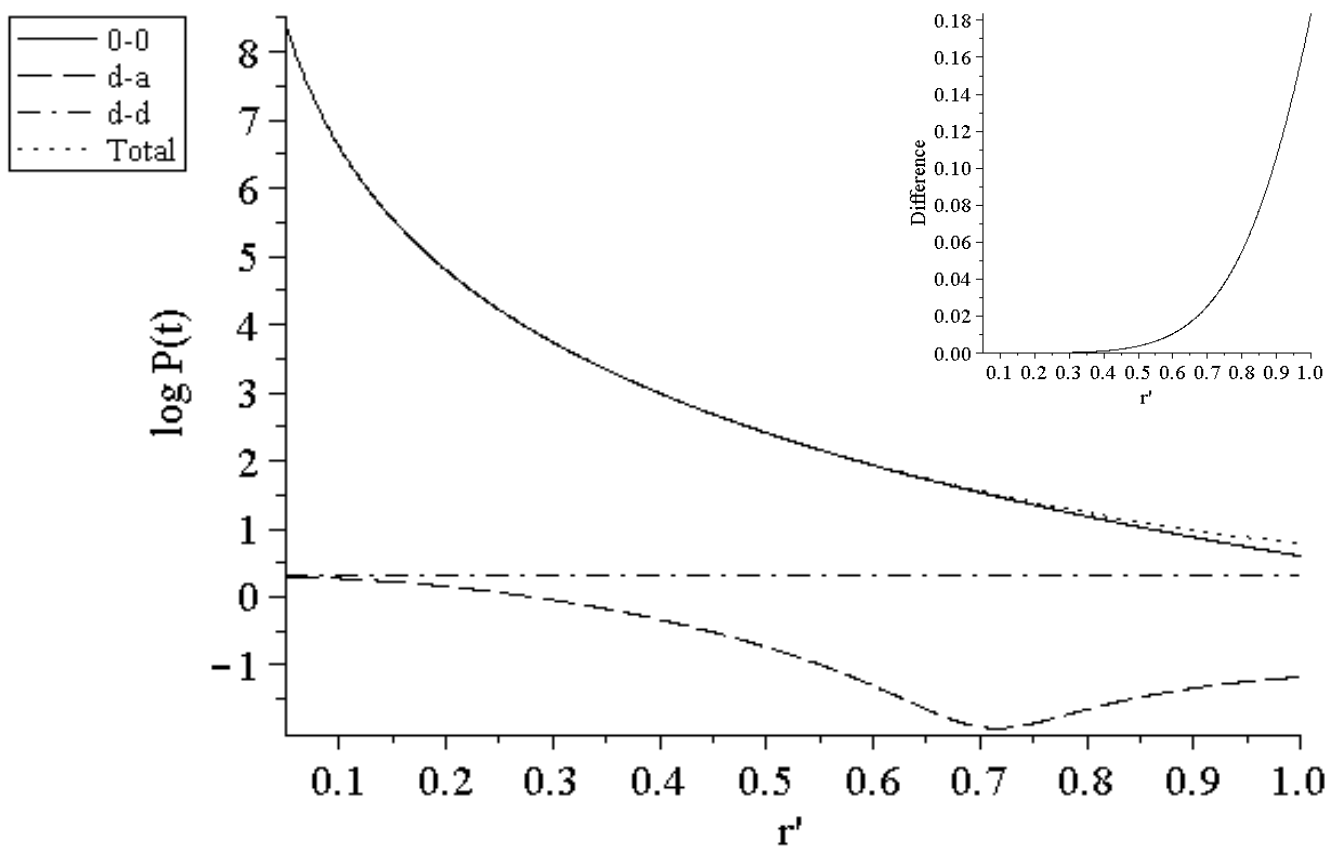

Figure 4. Plot of $\log P(t)$, the time-dependent probability, against the aspect ratio, $r^{\prime}$, for optical transfer from an excited molecule in the donor linear array to the required destination in the acceptor linear array (0-0); also depicted are the 'cross-talk' probabilities for transfer to another molecule in either the acceptor (d-a) or the donor (d-d) array, and the sum of all three transfer possibilities (Total). Insert: difference between 0-0 and the sum for various $r^{\prime}$, where on the ordinate axis each 0.01 difference corresponds to $2.3 \%$ loss. 
identical to (4) with $u$ set as null. Inserting (7) into (5) gives the expression;

$$
P(t)=\frac{K C^{2} J^{\prime}\left(u^{2}-2 r^{\prime 2}\right)^{2}}{l^{6}\left(u^{2}+r^{\prime 2}\right)^{5}} \sin ^{4} \phi \cos ^{2} \theta \sin ^{2} \theta
$$

Next to be considered is the case of energy transfer from the initially excited molecule to another within the donor array. Here the corresponding expression is analogous to equation (8), but with $r^{\prime}$ equal to zero. The subsequent aim is to compare and contrast the probability of energy relocation to the excited donor's partner in the acceptor array, against the sum of probabilities for transfer to all other molecules within both arrays. This is achieved by a graphical depiction of $P(t)$, with arbitrary units, for different transfer destinations as shown in Fig. 4. In this representation, energy transfer outside the range $u= \pm 10$ is negligible. Calculations are performed with $\Delta \mathrm{E}=3.3 \times 10^{-20} \mathrm{~J},|\boldsymbol{\mu}|=1 \times 10^{-29} \mathrm{C} \mathrm{m}, \phi=90^{\circ}$ and $\theta=45^{\circ}$. As the different curves within Fig. 4 illustrate, the transfer of energy from the excited donor to the corresponding molecule in the acceptor array, i.e. migration between the pair at $u=0$, greatly dominates all other transfer possibilities combined for an aspect ratio up to 0.5 . In fact no less than $98.9 \%$ of the total excitation is transported to the required destination. Conversely, on changing the aspect ratio to $r^{\prime}=1$, the amount of cross-talk to other molecules becomes very significant, with $35.4 \%$ of the desired transfer lost (see Fig. 4, insert). Notably (unlike the following two-dimensional systems) the destination of the initial excitation is not dependent on the laser intensity. Results from this section clearly show that, with favorable $r^{\prime}$ values, linear arrays are tenable as components in an all-optical switch. Nonetheless, a practically more useful solution will be based on two-dimensional arrays, the subject of the next two sections.

\section{TWO-DIMENSIONAL SQUARE-LATTICE ARRAYS}

A system more feasible as an all-optical switch involves a pair of two-dimensional square-lattice arrays (Fig. 3). To develop theory for this system, the linear arrays of the previous section are expanded into two dimensions - so that each molecule within an array is now labeled $(u, v)$, where $u$ and $v$ are integers, and also $\mathbf{R}=u \hat{\mathbf{i}}+v \hat{\mathbf{j}}+r \hat{\mathbf{k}}$. In this case RET is no longer always null, and may occur between the donor and off-axis molecules, ${ }^{1}$ i.e. where $u \neq 0$ and $v \neq 0$. To find an expression for $P(t)$ for this system the following equation, as determined in earlier RET work, ${ }^{17}$ is required;

$$
M_{f i}^{(2)} \bar{M}_{f i}^{(2)}=\left(\frac{\left|\boldsymbol{\mu}_{A}\right|^{2}\left|\boldsymbol{\mu}_{B}\right|^{2} \kappa^{2}}{16 \pi^{2} \varepsilon_{0}^{2} R^{6}}\right),
$$

where $\kappa=\cos \theta-3 \cos \phi \cos \gamma$ (with $\theta$ the angle between $\left|\boldsymbol{\mu}_{A}\right|$ and $\left|\boldsymbol{\mu}_{B}\right|$, and $\phi, \gamma$ the angles between $\mathbf{R}$ and $\left|\boldsymbol{\mu}_{A}\right|$, $\left|\boldsymbol{\mu}_{B}\right|$ respectively). In application to this system equation (9) becomes;

$$
\left|M_{f i}^{(2)}\right|^{2}=\left(\frac{|\boldsymbol{\mu}|^{4}}{16 \pi^{2} \varepsilon_{0}^{2} l^{6}}\right)\left(\frac{9 u^{2} v^{2}}{\left(u^{2}+v^{2}+r^{\prime 2}\right)^{5}}\right),
$$

since $\kappa=-3 u v /\left(u^{2}+v^{2}+r^{\prime 2}\right)$. The transfer contribution due to OCRET is determined analogously, to give;

$$
M_{f i}^{(4)}=\left(\frac{-n \hbar c k|\mu|^{4}}{8 \pi \varepsilon_{0}^{2} l^{3} V \Delta E_{A} \Delta E_{B}}\right)\left(\frac{u^{2}+v^{2}-2 r^{\prime 2}}{\left(u^{2}+v^{2}+r^{\prime 2}\right)^{\frac{5}{2}}}\right) \sin ^{2} \phi \cos \theta \sin \theta
$$




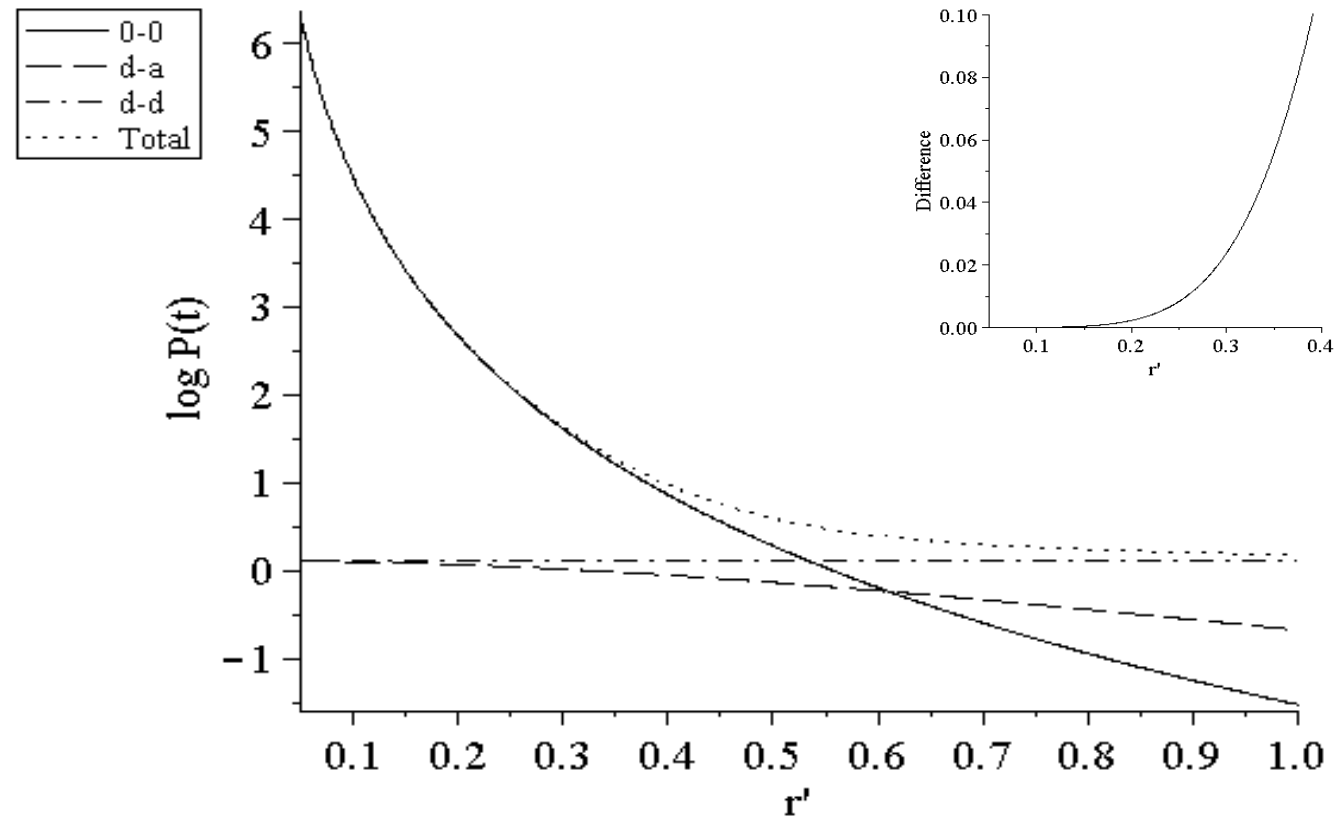

Figure 5. Graph illustrating $\log P(t)$ against $r^{\prime}$ for pair of two-dimensional square arrays. Here, the intensity $I$ of the input laser is $1 \times 10^{12} \mathrm{~W} \mathrm{~cm}^{-2}$ and the key is that of Fig. 4 .

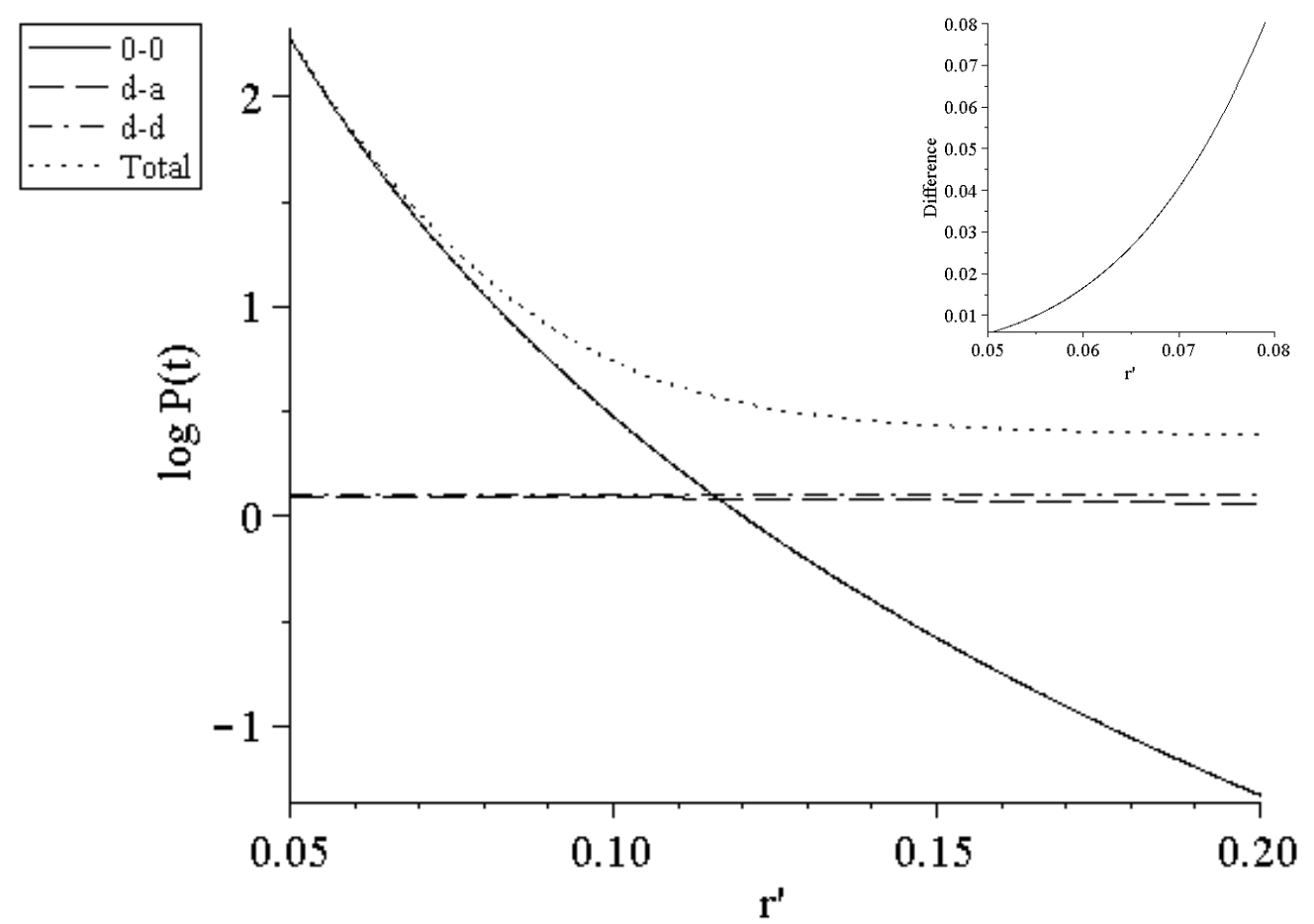

Figure 6. Graph as Fig. 5, but for $I=1 \times 10^{10} \mathrm{~W} \mathrm{~cm}^{-2}$. 
as $\kappa^{\prime}=\left(u^{2}+v^{2}-2 r^{\prime 2}\right) /\left(u^{2}+v^{2}+r^{\prime 2}\right)$. Employing equations (10) and (11) with (5), the following is determined;

$$
\begin{aligned}
P(t) & =\frac{K}{l^{6}\left(u^{2}+v^{2}+r^{\prime 2}\right)^{5}}\left[9 u^{2} v^{2} t+6 C J u v\left(u^{2}+v^{2}-2 r^{\prime 2}\right) \sin ^{2} \phi \cos \theta \sin \theta\right. \\
& \left.+C^{2} J^{\prime}\left(u^{2}+v^{2}-2 r^{\prime 2}\right)^{2} \sin ^{4} \phi \cos ^{2} \theta \sin ^{2} \theta\right]
\end{aligned}
$$

where the fluence $J=\int_{0}^{t} I(t) \mathrm{d} t$. As earlier for linear arrays, a graphical depiction of $P(t)$ for various transfer destinations (see Figs 5 and 6 ) is used to analyze the probability of energy transfer to the excited donor's partner in the acceptor array against the summed probabilities for transportation of the energy to all other molecules. Calculations are performed with the same parameter values as previously employed. The laser intensity, which for simplicity is assumed time-independent, takes the value of $1 \times 10^{12} \mathrm{~W} \mathrm{~cm}^{-2}$ and $1 \times 10^{10} \mathrm{~W} \mathrm{~cm}^{-2}$, for Figs 5 and 6 respectively. From a comparison of these graphical representations, it is clear that intensity is a major contributing factor in determining the destination of the donor excitation. To achieve transfer losses less than 5\% the aspect ratio, $r^{\prime}$, can be little over 0.3 for $I=1 \times 10^{12} \mathrm{~W} \mathrm{~cm}^{-2}$, or 0.06 for $1 \times 10^{10} \mathrm{~W} \mathrm{~cm}^{-2}$. These $r^{\prime}$ values are both practicable, but in the latter case may be most readily implemented through an expansion of the lattice constant, compromising the miniature dimensions of a device. The next section will determine whether these values can be improved upon, by examining two-dimensional hexagonal-lattice arrays as alternative components for realization as an all-optical switch.

\section{TWO-DIMENSIONAL HEXAGONAL-LATTICE ARRAYS}

In the investigation of an array of two-dimensional hexagonal-lattices, it is convenient to choose a coordinate system where $\mathbf{R}=u l \hat{\mathbf{a}}_{1}+v l \hat{\mathbf{a}}_{2}+r \hat{\mathbf{k}}$, which is converted into Cartesian coordinates so that;

$$
\mathbf{R}=\frac{\sqrt{3} l}{2}(u-v) \hat{\mathbf{i}}+\frac{l}{2}(u+v) \hat{\mathbf{j}}+r \hat{\mathbf{k}}
$$

For a pair of hexagonal-lattices, RET is possible from the donor to all other molecules, except where $u=v$ or $u=-v$, as is determined from the following expression;

$$
\left|M_{f i}^{(2)}\right|^{2}=\left(\frac{108|\boldsymbol{\mu}|^{4}}{\pi^{2} \varepsilon_{0}^{2} l^{6}}\right)\left(\frac{(u-v)^{2}(u+v)^{2}}{\left(3(u-v)^{2}+(u+v)^{2}+4 r^{\prime 2}\right)^{5}}\right)
$$

and $\kappa=-3 \sqrt{3}(u-v)(u+v) /\left(3(u-v)^{2}+(u+v)^{2}+4 r^{\prime 2}\right)$. The transfer contribution due to OCRET is resolved by replicating the previous method, and hence;

$$
M_{f i}^{(4)}=\left(\frac{-n \hbar c k|\boldsymbol{\mu}|^{4}}{\pi \varepsilon_{0}^{2} l^{3} V \Delta E_{A} \Delta E_{B}}\right)\left(\frac{3(u-v)^{2}+(u+v)^{2}+r^{\prime 2}}{\left(3(u-v)^{2}+(u+v)^{2}+4 r^{\prime 2}\right)^{\frac{5}{2}}}\right) \sin ^{2} \phi \cos \theta \sin \theta
$$




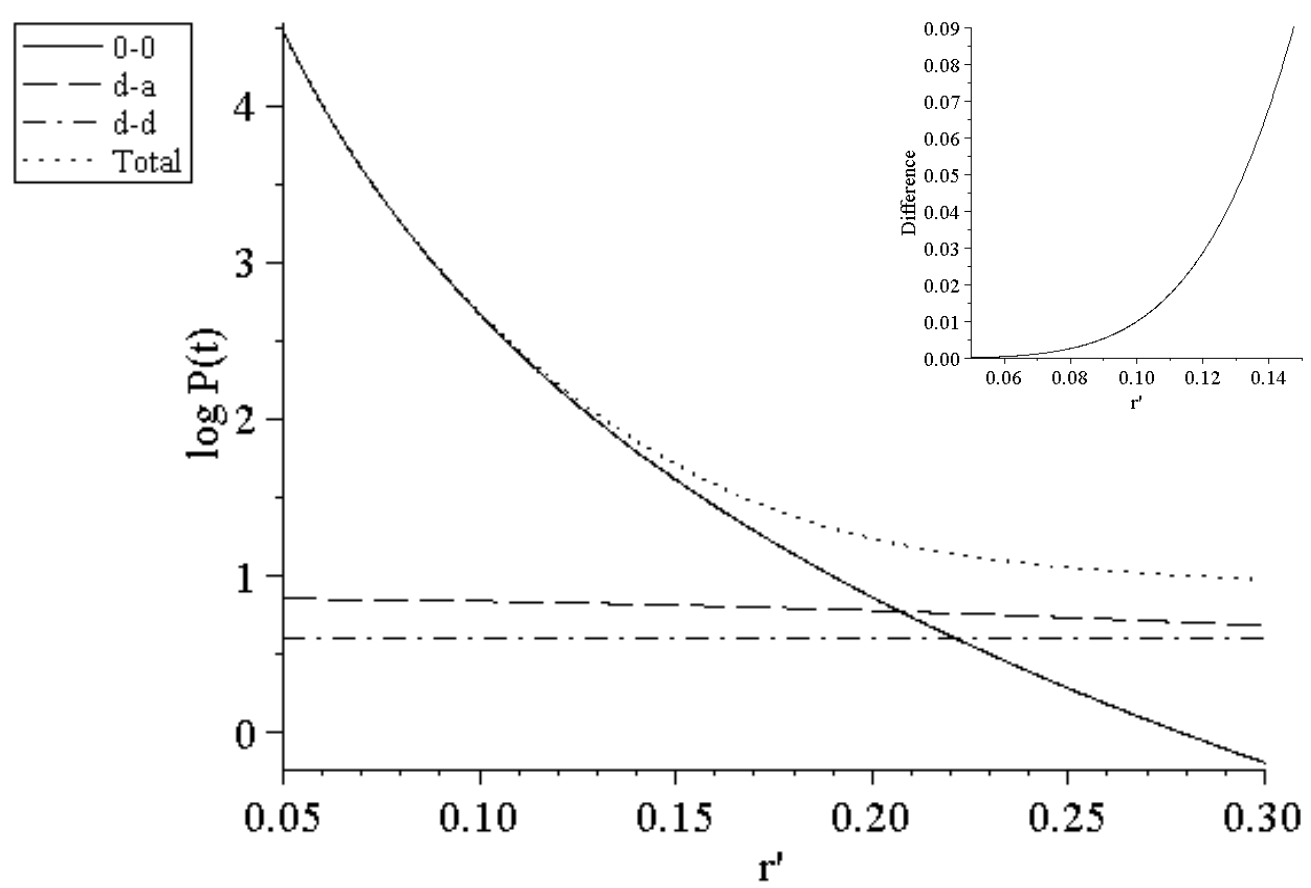

Figure 7. Graph illustrating $\log P(t)$ against $r^{\prime}$ for pair of two-dimensional hexagonal arrays. Here, the intensity $I$ of the input laser is $1 \times 10^{12} \mathrm{~W} \mathrm{~cm}^{-2}$ and key is given by Fig. 4 .

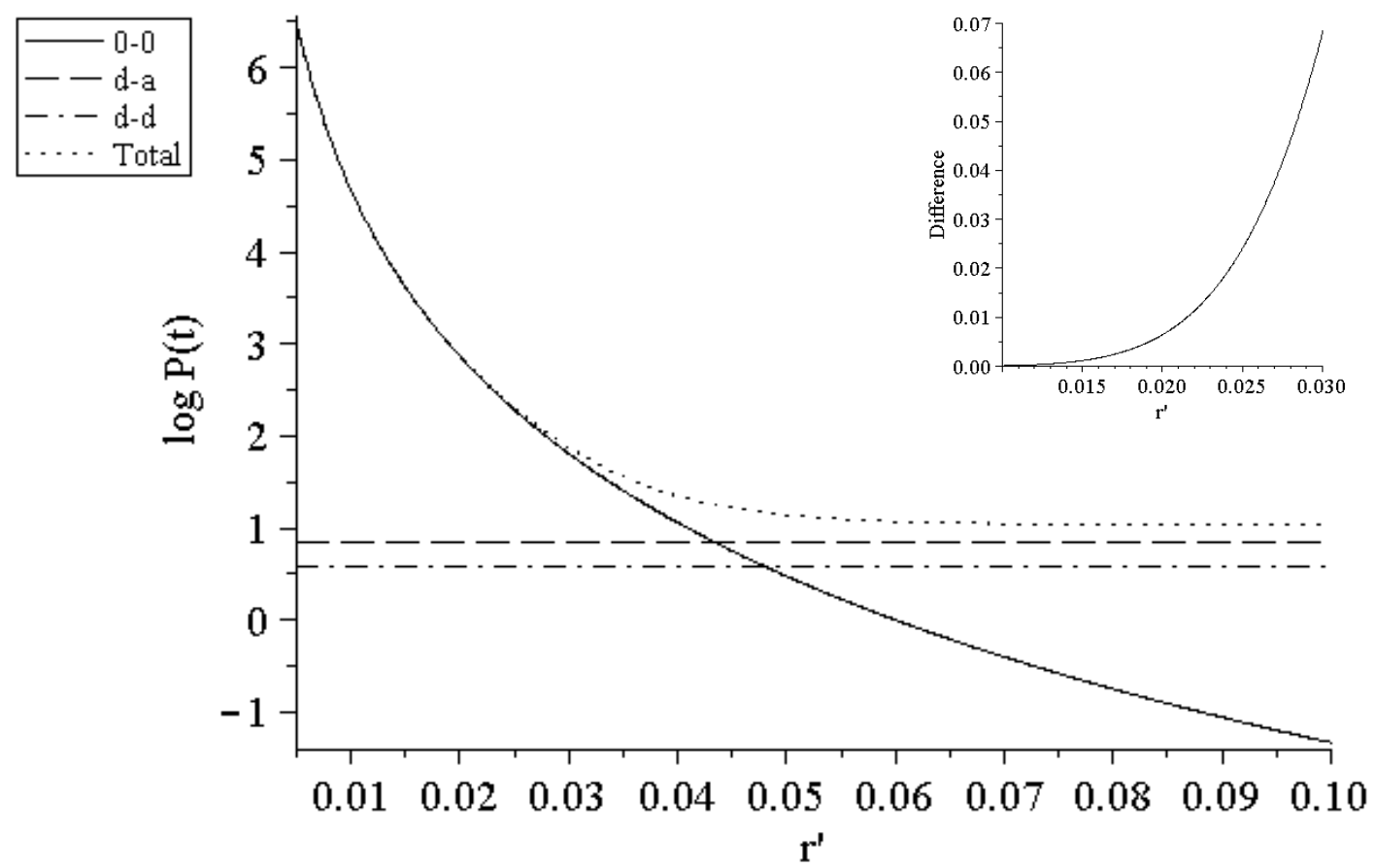

Figure 8. Graph as Fig. 7, but for $I=1 \times 10^{10} \mathrm{~W} \mathrm{~cm}^{-2}$. 
since $\kappa^{\prime}=\left(3(u-v)^{2}+(u+v)^{2}+r^{\prime 2}\right) /\left(3(u-v)^{2}+(u+v)^{2}+4 r^{\prime 2}\right)$. Through use of (14) and (15), with (5), the following result is found;

$$
\begin{aligned}
P(t) & =\frac{K}{l^{6}}\left[1728(u-v)^{2}(u+v)^{2} t+384 \sqrt{3} C J(u-v)(u+v)\left(3(u-v)^{2}+(u+v)^{2}+r^{\prime 2}\right) \sin ^{2} \phi \cos \theta \sin \theta\right. \\
& \left.+64 C^{2} J^{\prime}\left(3(u-v)^{2}+(u+v)^{2}+r^{\prime 2}\right)^{2} \sin ^{4} \phi \cos ^{2} \theta \sin ^{2} \theta\right]\left(3(u-v)^{2}+(u+v)^{2}+4 r^{\prime 2}\right)^{-5} .
\end{aligned}
$$

In the same manner as previously, various plots of $P(t)$ are constructed (Figs 7 and 8). On comparing these graphs it again transpires that the laser intensity plays a major role, as earlier for the square-lattice array system. To achieve transfer losses less than $5 \%$ in this case, $r^{\prime}$ cannot be much greater than 0.11 for $I=1 \times 10^{12} \mathrm{~W} \mathrm{~cm}^{-2}$, or 0.025 for $1 \times 10^{10}$ $\mathrm{W} \mathrm{cm}{ }^{-2}$ - values that are not favorable in comparison to the previous two-dimensional arrays system. This is explained by the fact that RET does not contribute to the transportation of excitation to the desired location, i.e. with $u=0$ and $v=0$ the first two terms of equation (16) are null, while all other potential destinations contain this RET contribution except where $u=v$ or $u=-v$.

\section{CONCLUSION}

This paper has investigated an all-optical nonlinear process that has potential for a variety of device implementations. The distinct features of several potentially fabricated nano-array structures have been analyzed in detail, and their relative efficiencies and practicality critically assessed. One-dimensional linear-lattice arrays prove the most efficient for controlling the throughput of excitation from initially excited donor species to counterpart acceptors. Two-dimensional arrays are more practically useful, in terms of the trade-off with nanoscale compactness, and square-lattice arrays represent a particularly favorable structure. Hexagonally close-packed arrays, despite their more efficient planar packing, prove less efficient through admitting a greater degree of information loss through cross-talk; the result is a convenient vindication of the square-lattice arrangement, which also has the advantage of simpler construction. In practice the layered fabrication of any such structure could be expedited by inclusion of a dielectric material between the arrays, which can be expected to further improve transfer efficiency. Ongoing research will involve an examination of the effects on transfer efficiency due to; the input of dual incident laser beams of different frequencies, the initial excitation of various molecules within the donor array, and a detailed analysis of other losses (e.g. through fluorescence). There are obvious switching, logic gate and transistor attributes to be explored, but the aspect that might hold the greatest promise for optical communications and data transmission is the capacity of such a system to act as an optical buffer.

\section{ACKNOWLEDGEMENTS}

We wish to thank the Leverhulme Trust for providing the financial support for this research.

\section{REFERENCES}

1. D. L. Andrews and R. G. Crisp, “Optically activated energy transfer: array implementation”, J. Opt. A 8, pp. S106-S112, 2006.

2. D. L. Andrews and R. G. Crisp, “Theory of directed electronic energy transfer”, J. Fluoresc. 16, pp. 191-199, 2006.

3. R. G. Crisp and D. L. Andrews, "Optical switching in nano-arrays: Transistor action through directed energy transfer”, Proc. SPIE, 61951I, 2006.

4. Resonance Energy Transfer, eds D. L. Andrews and A. A. Demidov (Wiley, Chichester, 1999).

5. P. Allcock, R. D. Jenkins and D. L. Andrews, "Laser assisted resonance energy transfer", Chem. Phys. Lett. 301, pp. 228-234, 1999. 
6. P. Allcock, R. D. Jenkins and D. L. Andrews, "Laser-assisted resonance-energy transfer", Phys. Rev. A 61, 023812, 2000.

7. D. L. Andrews, R. G. Crisp and S. Li, "Single and dual beam optical switching of resonance energy transfer", J. Chem. Phys. 127, 174702, 2007.

8. D. L. Andrews and D. S. Bradshaw, "Virtual photons, dipole fields and energy transfer: a quantum electrodynamical approach”, Eur. J. Phys. 25, pp. 845-858, 2004.

9. D. L. Andrews, S. Naguleswaran and G. E. Stedman, "Phenomenological damping of nonlinear-optical response tensors", Phys. Rev. A 57, pp. 4925-4929, 1998.

10. D. L. Andrews, L. C. D. Romero and G. E. Stedman, "Polarizability and the resonance scattering of light: Damping sign issues”, Phys. Rev. A 67, 055801, 2003.

11. P. W. Milonni and R. W. Boyd. "Influence of radiative damping on the optical-frequency susceptibility", Phys. Rev. A 69, 023814, 2004.

12. R. Loudon and S. M. Barnett, “Theory of the linear polarizability of a two-level atom”, J. Phys. B 39, pp. S555S563, 2006.

13. P. R. Berman, R. W. Boyd and P. W. Milonni, "Polarizability and the optical theorem for a two-level atom with radiative broadening", Phys. Rev. A 74, 053816, 2006.

14. S. Mukamel, "Causal versus noncausal description of nonlinear wave mixing: Resolving the damping-sign controversy”, Phys. Rev. A 76, 021803, 2007.

15. I. Bialynicki-Birula and T. Sowinski, arXiv.org:0705.2121 [quant-ph].

16. E. Fermi, Nuclear Physics (Chicago, University of Chicago Press, 1950).

17. G. J. Daniels, R. D. Jenkins, D. S. Bradshaw and D. L. Andrews, "Resonance energy transfer: The unified theory revisited", J. Chem. Phys. 119, pp. 2264-2274, 2003. 